\title{
Porokeratosis with an Invasive Squamous Cell Carcinoma: A Case Report and Review of Literature
}

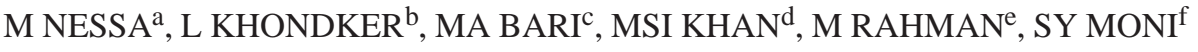

\begin{abstract}
Summary:
Porokeratosis is a clonal disorder of epidermal keratinization, which is characterized by hyperkeratotic papules or plaques that are surrounded by a thread-like elevated border. The histopathologic hallmark of porokeratosis is the cornoid lamella, which is a thin column of parakeratosis that overlies a thin or absent granular layer and that corresponds to the raised, hyperkeratotic border. Porokeratosis has five clinical types and malignant degeneration has been described in all forms of porokeratosis. We report a forty five year old farmer with a
\end{abstract}

\section{Introduction:}

Porokeratosis is a clonal disorder of keratinization characterized by one or more atrophic patches surrounded by a clinically and histologically distinct ridge-like border called 2 cornoid lamella2 . ${ }^{1,2}$ Five major clinical variants are recognized - classical or plaque type porokeratosis of Mibelli (PM), disseminated superficial actinic porokeratosis (DSAP), linear porokeratosis (LP), porokeratosis palmaris et plantaris disseminata (PPPD) and punctate porokeratosis (PP). ${ }^{1,3}$

a. Dr Moriom Nessa, Assistant Professor, Dept of Dermatology and Venereology, Islami Bank Medical College Hospital, Nawdapara, Rajshahi.

b. Dr. Lubna Khondker, Assistant Professor, Department of Dermatology and Venereology, Bangabandhu Sheikh Mujib Medical University (BSMMU), Shahbag, Dhaka, Bangladesh.

c. Dr. M A Bari MS (Urology), Rajshahi Medical College Hospital, Rajshahi.

d. Lt Col (Dr) Md Shirajul Islam Khan, Graded Specialist in Dermatology and Venereology, Combined Military Hospital (CMH), Dhaka cantonment, Dhaka.

e. Dr. Moksedur Rahman, Junior Consultant, Department of Dermatology and Venereology, Upazila Health Complex, Rajshahi.

f. Dr. Sabiha Yeasmin Moni, Rajshahi Medical College Hospital, Rajshahi.

Address of Correspondence: Dr. Lubna Khondker, Assistant Professor of Dermatology, Room-209(B), $2^{\text {nd }}$ floor, Block-B, Bangabandhu Sheikh Mujib Medical University (BSMMU), Shahbag, Dhaka. Mobile- 01552370429; E-mail: lubna_derma @yahoo.com

Received: 23 June, 2012

Accepted: 3 June, 2013 large plaque in chest for 30 years and multiple nodules within the large plaque for 2 years. A section of skin from margin of the plaque reveals histopathological features of porokeratosis and section from nodules reveals an invasive squamous cell carcinoma. To the best of our knowledge, this is the first reported case of porokeratosis transformed to squamous cell carcinoma on Bangladesh.

Key words: Porokeratosis, invasive squamous cell carcinoma.

(J Banagladesh Coll Phys Surg 2014; 32: 31-36)

Distinction between these types is made purely on clinical criteria. ${ }^{4}$ There are reports of more than one type of porokeratosis developing in the same patient. ${ }^{5}$ The most common form of porokeratosis is DSAP, typical lesions have symmetric distribution and usually affects sun-exposed areas. ${ }^{6}$ The etiology of porokeratosis remains unclear. Malignant degeneration has been reported in all forms of porokeratosis, with a reported incidence of 7.5 to 11 percent. $^{7}$ The occurrence of malignancies in porokeratotic lesions is clinical evidence of the pre-cancerous nature of this disease. ${ }^{8}$ Over-expression of p53 in lesional epidermis may play a role in malignant transformation. In addition, DNA flow cytometry analysis has demonstrated abnormal DNA ploidy in the lesional epidermis of patients with different types of porokeratosis. The most commonly associated malignant conditions with porokeratosis are Bowen disease, squamous-cell carcinoma (SCC), and, rarely, basal cell carcinoma. The greatest risk is attributed to large lesions, long duration, and linear-type lesions. ${ }^{9}$ Squamous cell carcinoma arising in the classic type of porokeratosis of Mibelli is well-documented, but there are only a few reports of squamous cell carcinoma in DSAP. ${ }^{10}$ There is no definitive treatment for Porokeratosis. Porokeratosis does not usually need treatment, but in some cases, treatment is necessary due to potential for progression to a malignancy and for 
cosmetic purposes. ${ }^{11}$ Attempts to treat with topical, systemic, and intralesional glucocorticoids; topical 5fluorouracil; phototherapy; and keratolytics have yielded only marginal benefits. Surgical modalities, such as cryotherapy, shave excision, curettage, linear excision, and dermabrasion have been used to treat small lesions with variable success. It has been suggested that oral retinoids may reduce the risk of malignant transformation. Relapses are common within weeks to months after cessation of oral retinoid therapy. ${ }^{12}$

\section{Case report:}

A 45 year old farmer from Porsha, Nowgaon reported to department of Dermatology and venereology, Islami Bank Medical College Hospital, Rajshahi, with a large plaque in chest for 30 years and multiple nodules within the large plaque for 2 years. According to the statement of the patient, he was alright 30 years back, and then he first developed a papule in the left chest just under the left nipple. Then it spreads slowly towards periphery and become depressed centrally with some scaly lesion in the centre, later it becomes serpiginous large well defined plaque, surrounded by a keratotic wall. This wall is grayish and surmounted by a tiny groove along its summit. Now left sided chest, left abdomen and left back is occupied by a large plaque. First, it was symptom less and he gives no history of burning, itching or pain. In the meantime he went to some local doctors and he took some medicine from doctors but failed to cure. He is a farmer and he works in the sunlight in the field. He gave history of trauma in the patch 6 years back, at the time of catching fish. After 28 years of that lesion, he noticed multiple nodules growing within the plaque, some are small and one is large nodule. Large nodule is situated below the left nipple, at the site of origin. The nodule is everted, cauliflower shaped with some exudation within centre. Now patient gives history of itching and burning. Patient is non-diabetic and normotensive. He gives no history of taking chemotherapy, PUVA, organ transplantation or no abnormality is detected in the mucous membrane, hair and nail. Nothing contributory history of drug, family or personal history. On examination, the plaque is surrounded by a keratotic border. The nodule is large, everted, cauliflower shaped and exudative in nature.

All the hematological( TC, DC,ESR, Hb\%) and biochemical(Random blood sugar, serum ALT, serum creatinine) parameter was within normal limit. From two sites of skin, biopsy material was taken, one from margin of the plaque and another from large nodule and sent for histopathological examination. A section of skin from margin of the plaque reveals mild hyperkeratosis and a shallow depression containing columns of parakeratotic cells. Epidermis below this area shows dyskeratotic changes and the dermis reveals a moderate infiltration of chronic inflammatory cells. These features are confirmatory of porokeratosis, on histo-pathologically, though classical or plaque type porokeratosis variety on clinically. Section from nodules reveals an invasive squamous cell carcinoma. The tumor is well differentiated. It has invaded the subcutis. The deep margin shows presence of tumor (Grade-I). Patient was treated by topical steroid and retinoid and advised for excision of nodule.

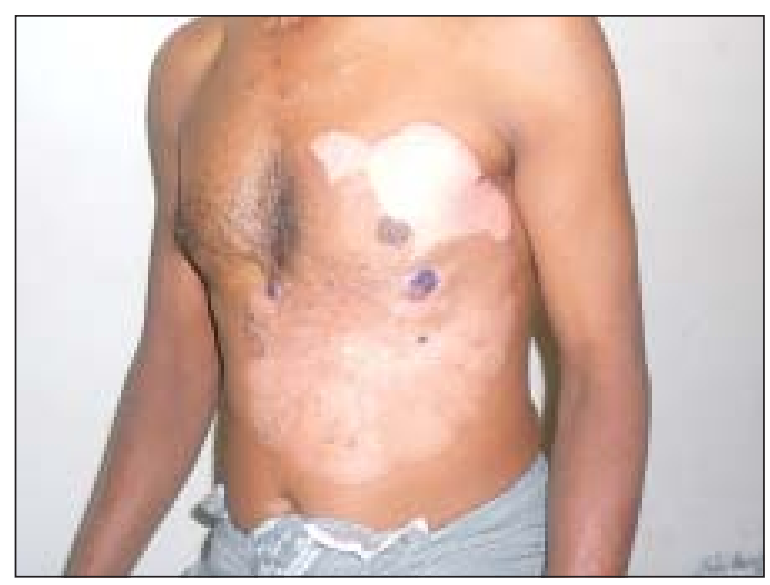

Fig.-1: Photograph of the patient with a large well defined plaque, surrounded by a keratotic wall in the chest and multiple nodules within the large plaque.

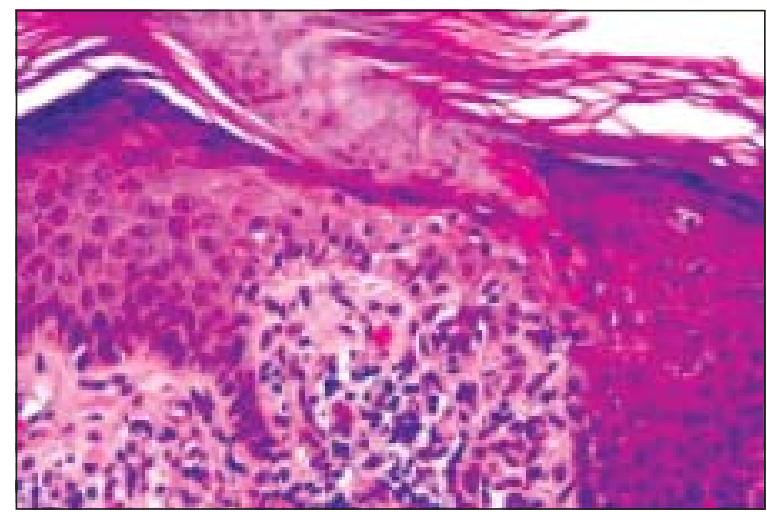

Fig.-2: Photograph of histopathological finding from margin of the plaque showing - cornoid lamella of porokeratosis. 


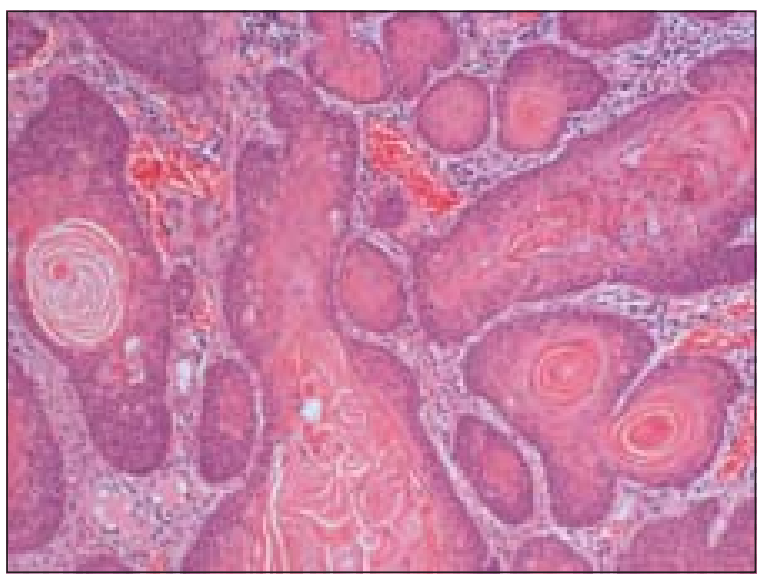

Fig.-3: Photograph of histopathological finding from large nodule showing Squamous cell carcinoma (SCC)

\section{Discussion:}

Sengupta Sujata et al reported a case of 40-year-old farmer (case 1) with asymptomatic skin lesions over the extremities, abdomen, groins, palms and soles for the past ten years. This case was reported in the department of Dermatology, R.K.M Seva Pratisthan and Vivekananda Institute of Medical Sciences, Kolkata, India. Since the last 6 months, he noted the appearance of multiple raised mildly itchy but painless growths over the pre-existing lesions in the groins. His family history was not suggestive of a similar disease. He denied an exposure to radiation and there was no history of highrisk sexual practices. No evidence of immunosuppression was apparent. On examination, numerous small well-defined hyperpigmented plaques, some of which were more than $10 \mathrm{~cm}$ in diameter, were seen. These giant lesions were present in the abdomen, thighs and soles. The involvement of the palms and soles was prominent. Individual plaques had an atrophic center with a prominent border that was traversed by a threadlike groove. In the inguinal region on both sides there were multiple erythematous papulonodular lesions of varying sizes, which bled on manipulation. The largest of them was $5 \mathrm{~cm} \times 3 \mathrm{~cm} \times 2 \mathrm{~cm}$. A few inguinal lymph nodes palpable were less than $0.5 \mathrm{~cm}$ in size, soft to firm, not tender and mobile. No other lymph node was palpable. The rest of the cutaneous and systemic examination was normal. Routine blood tests, biochemical examinations, X-ray of the chest were normal. VDRL and ELISA for HIV were nonreactive. Histology from the edge of a flat lesion showed a parakeratotic column (cornoid lamella) in the epidermal invagination with absence of the underlying granular layer. A non-specific perivascular infiltrate of chronic inflammatory cells was seen in the dermis. These findings were consistent with porokeratosis. Fine needle aspiration and cytology (FNAC) from two contralateral inguinal nodules showed atypical cells and histopathology revealed features of a well-differentiated SCC in both. Ultrasonography of the abdomen showed no organomegaly, lymph node enlargement or any evidence of metastasis. A CT-guided FNAC of the inguinal and femoral lymph nodes showed reactive hyperplasia without any cellular atypia. The diagnosis of multicentric SCC arising from PPPD and giant porokeratosis was reached. ${ }^{13}$

Nilendu Sarma et al reported a 51-year-old male (case 2) and his 23-year-old son with multiple, well-defined, annular oval, polycyclic or irregular shaped lesions with raised margins in the Department of Dermatology, NRS Medical College, Kolkata, India. All the lesions were dark brown and were widely distributed both in the exposed as well as the covered areas. They were dry, palpable, and the margins were elevated compared with the central area. Along the margin, there was a prominent deep furrow. Most of the lesions measured 4-6 cm in their longest dimension and were slightly larger in size in the father. The father had some additional features. Many lesions, even smaller ones, showed a tendency towards development of gross hypertrophy in the margin with well formed horns in some. The central part of the lesions showed clinically normal skin in most lesions. Some lesions also appeared warty with a prominent break on the entire surface. In the father, there was a large ulcer $(8 \times 9 \mathrm{~cm})$ over both the buttock and perianal areas with predominant lateral extensions towards the patient's left side (at 3 to 6 o' clock) and towards the patient's right side (9 to11 o' clock). The ulcer was surrounded by typical lesions with hyperkeratotic margin. The ulcer involved the anal margin exposing the mucosal layer that appeared mildly prolapsed. There were also multiple lesions scattered over the buttocks; their sizes were 3 to $6 \mathrm{~cm}$ and mostly oval in shape. Considering the clinical presentation, they diagnosed these cases as disseminated Mibelli's plaque type porokeratosis with multiple horns and an ulcer in the father. The father's grandmother, his both sons (including one who attended), and daughter had similar diseases. His father was asymptomatic until 33 years of 
age when he died of unknown cause. His mother did not have the same disease. All who had the disease started to develop the lesions during the age of 12-15 years. Histological examination of the lesion was done in the father from two sites; one from the margin of a well-defined hyperkeratotic lesion over the foot and another from the ulcer in the perianal region. Histology from the margin of clinically typical porokeratosis over the foot showed a column of severe hyperkeratosis and epidermal invagination filled with parakeratosis (coronoid lamella). The subepidermal stroma showed mild infiltration of lymphocytes. This histomorphological feature was consistent with our clinical diagnosis of porokeratosis.A histopathological examination of the ulcer in the perianal region showed atypical squamous cells in clusters and sheets with surface ulceration. The squamous cells showed moderate pleomorphism, hyperchromatic nuclei, and prominent nucleoli. Brisk mitosis and focal necrosis were observed. Keratin pearl and evidence of intracytoplasmic keratin were noted. On the basis of these histological features, a diagnosis of keratinizing squamous cell carcinoma was made. The tumor involved the anal canal reaching up to the squamo-columnar junction in its upper part. The anal sphincteric muscles were also infiltrated by the tumor. A histological examination of a skin lesion on the back from the son also showed features consistent with porokeratosis of Mibelli. Finally, the cases were confirmed as familial porokeratosis of Mibelli's type with malignant transformation to squamous cell carcinoma over the buttock involving the anus in the father. Unfortunately, the patients were lost during the work-up period even before any therapeutic intervention could be made. ${ }^{14}$

A 73-year-old woman (case 3) was referred to the Disorders of Keratinization Clinic at Bellevue Hospital Center 27 years ago with scaly lesions that began on her soles and progressively spread to her palms, legs, thighs, upper extremities, trunk, neck, and face. Her mucous membranes were spared. The papules on the palms and soles became increasingly hyperkeratotic and confluent into thick plaques. The palmoplantar lesions are painful due to pressure and interfere with ambulation and manual dexterity. Some of the other lesions are pruritic, especially in the summer and on exposure to heat. The patient has a history of atrial fibrillation that is treated with warfarin, dofetilide, and propranolol. She also has hyperlipidemia that is treated with atorvastatin. After an evaluation of bone density for retinoidassociated osteoporosis, treatment with alendronate was initiated. She has no known allergies to medications or family history of dermatologic diseases. She worked in a factory sewing cases for cosmetic products for more than 20 years although she retired in 2001. Histopathologic examination of a biopsy speciman from a lesion on her right upper thigh at the time of presentation confirmed the suspected clinical diagnosis. A biopsy specimen obtained in 2002 disclosed the same diagnosis. She was initially treated with isotretinoin daily, which resulted in a decrease in the number of lesions on the body and in the hyperkeratosis of the papules on the palms and soles. In 1985, the medication was changed to etretinate and this medication was even more effective in decreasing the number of lesions and the palmoplantar hyperkeratosis. In 1998, after removal of etretinate from the market, acitretin was initiated at a dose of $25 \mathrm{mg}$ daily, with similar clinical efficacy as the parent compound. Attempts to discontinue or decrease systemic retinoids have resulted in pain and pruritus that interfere with her quality of life. Conversely, attempts to increase the dose of acitretin have been limited by both cheilitis and systemic adverse effects, which include blackouts. Numerous adjunctive treatments to diminish hyperkeratosis have been tried; these include salicylic acid 6 percent gel, salicylic acid in 10 to 40 percent concentrations, and urea 20 to 50 percent with and without occlusion. These agents have facilitated debridement of the palms and soles, which has been routinely performed by the patient and periodically in the office. Prolonged treatments with topical imiquimod and fluorouracil neither produced improvement nor prevented formation of new lesions. Topical tretinoin had to be discontinued because of erythema and pruritus. Destructive modalities, which included 90 percent trichloracetic acid, cryotherapy, and electrodesiccation have been successful in treating individual lesions. Physical examination reveals innumerable 2-3 mm; grey, annular papules with well-demarcated, slightly raised, hyperkeratotic borders covered her face, chest, back, abdomen, and extremities. Lesions were present in both sun-exposed and sun-protected areas. On the palms and soles, there were numerous, dicrete, non-punctate lesions as well as a confluence of papules, some with elevated borders. Her mucous membranes were spared. 
A complete blood count with differential analysis and comprehensive metabolic panel were normal. Histopathological findings reveal focal thinning of the epidermis, with loss of the granular layer, and a discrete column of parakeratosis. 15

A 62-year-old male (case 4) presented with pruritic eruptions on sun-exposed portions of both forearms that had gradually increased in number over a period of 5 years. The lesions were exacerbated during the summer months. Along with these lesions, an erythematous, irregular, marginated, scaly, crusted plaque had developed on the right forearm 3 years earlier. There was no significant medical or family history.An irregular, marginated, erythematous plaque and multiple, brown, atrophic macules surrounded by well-demarcated, raised ridges on the right forearm. On physical examination, a $2 \times 3 \mathrm{~cm}$ erythematous, irregular, marginated, scaly, crusted plaque was noted on the right forearm. In addition, the patient had numerous annular, brown, atrophic, and symmetric macules surrounded by welldemarcated, raised ridges on extensor aspects of both forearms, which are characteristics of DSAP. Complete blood count, as well as liver and kidney function tests were all within normal limits. A skin biopsy specimen of the multiple, brown, annular lesions showed histologic changes of typical DSAP. There was a cornoid lamella composed of a column of parakeratosis with underlying hypogranulosis and perivascular lymphocytic infiltrations in the dermis localized beneath the cornoid lamella. A skin biopsy obtained from the erythematous plaque on the right forearm showed dysregulated keratinocytes with hyperchromatic, atypical nuclei, consistent with squamous cell carcinoma. A cornoid lamella was observed in the lesion of the squamous cell carcinoma. Biopsy specimen obtained from the erythematous plaque on the right arm. In epidermis, acanthosis and dysregulated keratinocytes with hyperchromatic, atypical nuclei are observed. A cornoid lamella composed of a column of parakeratosis is seen in the lesion. Positron emission tomographycomputed tomography revealed no evidence of distant metastasis. The squamous cell carcinoma was treated by total excision and split-thickness skin graft and radiotherapy. The patient is currently being treated with topical sunscreens. 16

Researchers in the department of Dermatology in the University of Tokushima, Japan reported that they have shown p53 over-expression immuno-histochemically in 14 of 17 porokeratotic specimens obtained from 14 lesions of nine cases, and in all six specimens of squamous cell carcinoma (SCC) arising on porokeratotic lesions of two cases. We screened mutations in exons 5 to 10 of the p53 gene in all these specimens by polymerase chain reaction-single strand conformation polymorphism analysis. Mutations of the p53 gene were detected in two of the six SCCs but not in any of the 17 porokeratotic specimens. These two mutations were $\mathrm{C}$ to $\mathrm{T}$ transitions at codons 146 and 175 in exon 5, which were a nonsense mutation at a dipyrimidine site and a missense mutation at a CG site, respectively. To our knowledge, neither of these mutations has been identified in skin cancers before. Our observations indicate that mutations of the 553 gene are not the major molecular etiology for porokeratosis, but are related to its skin carcinogenesis, and that p53 overexpression in porokeratosis is not due to p53 gene mutations. ${ }^{17}$

Christine Liang reported a 22-year-old man(case 5), presented to the Dermatology Clinic at Bellevue Hospital Center in July, 2008, with a two-year history of warts on the shaft of his penis, thighs, scrotum, and perianal area. An external biopsy report brought in by the patient had been interpreted as condyloma acuminata. The patient was treated at an outside clinic with podophyllin and cryotherapy and noted improvement of the perianal lesions but limited improvement of the lesions on the penis and scrotum. Past medical history was noncontributory. The patient was sexually active with both men and women and reported a history of unprotected sex. He denied a prior history of sexually transmitted diseases. The patient took no medications. A punch biopsy was obtained from a lesion on the scrotum. Physical Examination reveals that on the dorsal shaft of the penis were multiple, small, annular plaques with a thin, threadlike border. Numerous, arcuate, erythematous plaques with borders consisting of small erythematous papules were noted on the scrotum. On the left inguinal fold were multiple verrucous papules and plaques. The perianal area was clear. A rapid plasma reagin test was non-reactive. Urine DNA amplification testing for gonorrhea and chlamydia were negative. Within a hyperplastic epidermis there is a dell with hypogranulosis, dyskeratosis, and an overlying column of parakeratosis, which is consistent with a cornoid lamella. Beneath this is a band-like lymphohistiocytic infiltrate. ${ }^{18}$

A 25 year male (case 6 ) reported with skin lesions of 12 years duration. The lesions started as small keratotic papules over his perineum which spreaded peripherally very slowly and eventually formed well defined plaques surrounded by irregular keratotic border with atrophic center. The lesions gradually spreaded to penis, upper 
thighs, feet and a few lesions scattered over trunk, face and scalp. There was no history of contact with chemicals or any medications. The lesions were skin colored, hairless and of variable sizes. All relevant investigations were within normal limits. Fungal microscopy, fungal culture, montoux test, STS were negative. Skin biopsy for histopathological examination revealed features of consistence with porokeratosis Mibelli. Genetic analysis could not be done due to lack of facilities. From this case 1, their study was able to trace 9 other members in successive generations of his family with porokeratosis. The case 1(propositus) inherited the disease from his maternal grandfather. The individuals affected in 3 successive generations are 10 in numbers. Of the 10 cases, 9 were examined personally and had histological evidence of porokeratosis; the rest one was reliably reported by close relatives to have the disease. None of the cases eventually developed any cutaneous malignancy. Among the ten affected patients 5 were male and 5 were female. In generation- I, one out of two; In generation- II, two out of five; In generation- III, seven out of fourteen family members were affected. The age of affected members ranged from 18 to 17 years and the age of disease onset was between 12 to 17 years. In that study, authors could not find out any malignant degeneration in any case. Cases in 3 successive generations suggest that porokeratosis is a genetic disorder and not a simple dyskeratosis. ${ }^{19}$

\section{Conclusion:}

A case of malignant transformation from classical or plaque type porokeratosis is reported here. Malignant change has been reported in nearly all types of porokeratosis. A review of malignant transformation found that these changes were more frequent on nonexposed skin, in large porokeratosis lesions, and in patients who previously received radiation therapy. Therefore, long-term follow-up for porokeratosis lesions is reasonable and long-term follow-up studies are needed.

\section{References:}

1. Hanumanthayya K, Nagari S, Tophakhane R, Rathor R. Coexistence of disseminated superficial and giant porokeratosis of Mibelli with squamous cell carcinoma. Indian J Dermatol Venereol Leprol 2003; 69:296-7.

2. Curnow P, Foley P, Baker C. Multiple squamous cell carcinoma complicating linear porokeratosis. Aust J Dermatol 2003; 44:136-9.

3. Kaur S, Thami GP, Mohan H, Kanwar AJ. Co-existence of variants of porokeratosis: a case report and review of literature. J Dermatol 2002; 29:305-9.
4. Seishima M, Izumi T, Oyama Z, Maeda M. Squamous cell carcinoma arising from lesions of porokeratosis palmaris plantaris disseminata. Eur J Dermatol 2000; 10:478-80.

5. Kaur S. Co-existence of variants of porokeratosis: a case report and review of the literature. J Dermatol 2002; 29:305

6. Thomas C, Ogboli MI, Carr RA, Charles-Holmes R. Hypertrophic perianal porokeratosis in association with superficial actinic porokeratosis of the leg. Clin Exp Dermatol 2003; 28:676-7.

7. Liu P, Zhang S, Yao Q, Liu X, Wang X, Huang C, et al . Identification of a genetic locus for autosomal dominant disseminated superficial actinic porokeratosis on chromosome 1p31.3-p31.1. Hum Genet 2008;123:507-13.

8. Wei SC. Identification of a locus for porokeratosis palmaris et plantaris disseminata to a 6.9-cM region at chromosome 12q24·1-24·2. Br J Dermatol 2003;149:261

9. Darling MJ, Lambiase MC, Viernes J. Porokeratosis: a solitary oral lesion. J Drugs Dermatol 2005;4:89-91.

10. Jih M. Porokeratosis plantaris, palmaris, et disseminata. Dermatol Online J 2003 ; 9:34

11. Lin JH, Hsu MM, Sheu HM, Lee JY. Coexistence of three variants of porokeratosis with multiple squamous cell carcinomas arising from lesions of giant hyperkeratotic porokeratosis. J Eur Acad Dermatol Venereol 2006; 20:621-3.

12. Sengupta $\mathrm{S}$, et al. Multicentric squamous cell carcinoma over lesions of porokeratosis palmaris et plantaris disseminata and giant porokeratosis. Indian J Dermatol Venereol Leprol 2005; 71:414.

13. Sujata S, Jayanta KD, Asok G. Multicentric squamous cell carcinoma over lesions of porokeratosis palmaris et plantaris disseminata and giant porokeratosis. Indian Journal of Dermatology, Venereology and Leprology, 2005; 71(6): 414-416.

14. Sarma N, Boler AK, Bhattacharya SR. Familial disseminated plaque type porokeratosis with multiple horns and squamous cell carcinoma involving anal skin. Indian J Dermatol Venereol Leprol 2009;75:551

15. Rachael H, Rajni M, Miguel S, Jennifer A S. Porokeratosis plantaris, palmaris, et disseminate. Dermatology Online Journal 2010;16 (11): 22

16. Hyung Rae Lee, Tae Young Han, Sook-Ja Son, and June Hyunkyung Lee. Squamous Cell Carcinoma Developing within Lesions of Disseminated Superficial Actinic Porokeratosis. Ann Dermatol. 2011 November; 23(4): 536-538.

17. Ninomiya Y, Urano Y, Yoshimoto KY, Iwahana H, Sasaki S, Arase S, Itakura M.p53 gene mutation analysis in porokeratosis and porokeratosis-associated squamous cell carcinoma. Journal of Dermatological Science 1997; 14 ( 3): Pages 173-178

18. Liang C, Batra P, Patel R, Kamino H. Genital porokeratosis. Dermatology Online Journal 2009;15 (8): 23.

19. Shah MOR, Sadeque BMZ, Shahidullah M, Ullah QMM, Farooque MG, Ahmed Z. Familial Porokeratosis Mibelli -A report of 10 cases in 3 generations of a Family in Bangladesh. BAFM Journal 2000;26:15-17. 